\title{
openheart Body mass index as a risk factor for coronary events and mortality in patients with type 1 diabetes
}

\author{
Daniel Vestberg, ${ }^{1,2}$ Annika Rosengren, ${ }^{2}$ Katarina Eeg-Olofsson, ${ }^{2}$ Mervete Miftaraj, ${ }^{3}$ \\ Stefan Franzen, ${ }^{3}$ Ann-Marie Svensson, ${ }^{3}$ Marcus Lind ${ }^{1,2}$
}

To cite: Vestberg D, Rosengren A, Eeg-Olofsson $\mathrm{K}$, et al. Body mass index as a risk factor for coronary events and mortality in patients with type 1 diabetes. Open Heart 2018;5:e000727. doi:10.1136/ openhrt-2017-000727

Received 19 September 2017 Revised 2 December 2017 Accepted 29 December 2017

Check for updates

${ }^{1}$ Department of Medicine, NU-Hospital Organization, Trollhättan, Sweden

${ }^{2}$ Department of Molecular and Clinical Medicine, Institute of Medicine, Sahlgrenska Academy, University of Gothenburg, Gothenburg, Sweden

${ }^{3}$ Center of Registers in Region Västra Götaland, Goteborg, Sweden

Correspondence to Dr Daniel Vestberg; daniel. vestberg@vgregion.se

\section{ABSTRACT}

Objective To investigate the potential relationship between body mass index (BMI) and the risk for myocardial infarction and coronary death in patients with type 1 diabetes.

Methods We studied patients with type 1 diabetes included in the Swedish National Diabetes Registry during 2002-2004 and followed them until a discharge diagnosis for myocardial infarction, acute coronary event, death or until 31 December 2011. Cox regression was used to estimate relative risks.

Results In 17499 patients with type 1 diabetes (mean age 39.4 years; mean BMI $25.2 \mathrm{~kg} / \mathrm{m}^{2}$ ), 819 were diagnosed with myocardial infarction as a primary or secondary diagnosis during a mean follow-up of 8.5 years (maximum 9.9 years). Estimated with Cox regression, there was no significant effect of increased BMI on the risk of myocardial infarction (HR $1.4(95 \% \mathrm{Cl} 0.7$ to 2.5$)$ in the group with $\mathrm{BMI}>35 \mathrm{~kg} / \mathrm{m}^{2}$ compared with BMI $18.5-25 \mathrm{~kg} / \mathrm{m}^{2}$. There was no association between BMI and coronary mortality, acute coronary events or all-cause mortality after adjusting for other known risk factors. Underweight patients $\left(\mathrm{BMl}<18.5 \mathrm{~kg} / \mathrm{m}^{2}\right)$ had increased hazard for coronary (HR $5.0(95 \% \mathrm{Cl} 1.5$ to 16.9$))$ and allcause mortality (HR $5.4(95 \% \mathrm{Cl} 3.1$ to 9.6$))$ compared with BMI $18.5-25 \mathrm{~kg} / \mathrm{m}^{2}$

Conclusions Among patients with type 1 diabetes, increased BMI is not a significant independent risk factor for myocardial infarction or coronary death after adjustment for other risk factors. Low BMI (less than $18.5 \mathrm{~kg} / \mathrm{m}^{2}$ ) is associated with mortality from coronary or any cause.

\section{INTRODUCTION}

Cardiovascular disease (CVD) is a major cause of death and hospitalisation in Europe and other western countries. ${ }^{1}$ Type 1 diabetes is associated with a substantial excess risk for CVD compared with patients without diabetes, ${ }^{2-6}$ Although mortality has decreased, substantial excess mortality still exists among patients with type 1 diabetes compared with controls, ${ }^{7}$ and there is a strong association with glycaemic control. ${ }^{89}$ Overweight and obesity are well-known risk factors for CVD in the general population. ${ }^{10}$ Findings from previous studies on body mass

\section{Key questions}

What is already known about this subject? Increased body mass index (BMI) has been shown to be a risk factor for cardiovascular disease in the general population. Patients with type 1 diabetes have a nearly fourfold risk. Among patients with type 1 diabetes, there is conflicting evidence about the influence of BMI on risk for myocardial infarction.

What does this study add?

This study shows that BMI may not be an important risk factor for myocardial infarction and coronary death for patients with type 1 diabetes but indicates an increased risk for both cardiac and overall mortality in the group with low BMI $\left(<18.5 \mathrm{~kg} / \mathrm{m}^{2}\right)$.

How might this impact on clinical practice? Even if increased BMI does not lead to a higher risk for ischaemic heart disease, there is still a lot of evidence for weight control among persons with type 1 diabetes and especially underweight should be a warning sign for increased mortality.

index (BMI) and type 1 diabetes have been divergent. In the EURODIAB study, ${ }^{11} \mathrm{BMI}$ was not a significant risk factor for coronary heart disease (defined as physician diagnosis of either acute myocardial infarction (MI), angina pectoris or ECG abnormalities suggestive of probable ischemia), while two unadjusted analyses ${ }^{12}{ }^{13}$ found significantly higher mean baseline BMI among patients who subsequently developed CVD (non-fatal MI or stroke, death from CVD (MI or stroke), subclinical MI or angina pectoris, need for revascularisation with either angioplasty (percutaneous coronary intervention (PCI) ) or coronary artery bypass grafting $(\mathrm{CABG}))$. While a large meta-analysis of cause-specific mortality in the general population has shown increased mortality from ischaemic heart disease with increasing BMI, ${ }^{14}$ studies in patients with CVD have shown a protective effect of increased body weight on cardiovascular death, known as 
the 'obesity paradox'. ${ }^{15} 16$ Recently, a large meta-analysis in the general population showed a direct relationship between BMI and all-cause mortality, in contrast to previous studies. ${ }^{17}$ We previously found an increased risk for hospitalisation for heart failure ${ }^{2}$ among patients with type 1 diabetes compared with the general population. Additionally, elevated BMI seems to be a strong risk factor for the development of heart failure among patients with type 1 diabetes. ${ }^{18}$ Therefore, our primary objective was to determine the relationship between BMI and risk of MI and coronary death among patients with type 1 diabetes. Secondary objectives were to study the effect of BMI on major cardiac events and all-cause mortality.

\section{RESEARCH DESIGN AND METHODS}

We used a cohort based on data from the Swedish National Diabetes Registry (NDR), a quality assurance instrument in diabetes care with nationwide coverage for patients with diabetes over the age of 18 years. Patient data are continuously transmitted via electronic patient records from the clinic or registered directly online to the NDR. Each patient provides informed consent before inclusion. Data were linked to outcomes data from the Swedish hospital discharge and cause-specific death registries. Additional baseline information on level of education was collected through the Longitudinal Integration Database for Health Insurance and Labor Market Studies. 1920

Our cohort from the NDR consisted of all patients registered between January 2002 and December 2004 with type 1 diabetes and with at least one BMI measurement. In the NDR, patients with type 1 diabetes were receiving treatment with insulin only and age of onset 30 years or younger; these sample characteristics have previously been validated to be correct for a diagnosis of type 1 diabetes in $97 \%$ of cases. ${ }^{13}$

Our initial cohort consisted of 18312 patients 18 years or older. We then excluded patients over 80 years $(n=52)$ due to low estimated survival and potentially multiple causes of death in the death registries. After patients with previously known MI (ICD-10 (International classification of diseases, tenth revision) diagnosis of I20 or I21) were excluded $(\mathrm{n}=811), 17449$ patients remained. Study start was defined as the time of the patient's first registration in the NDR. The final study cohort consisted of 17449 patients who were followed until a primary or secondary endpoint, death or 31 December 2011.

Primary endpoints were MI (defined as ICD-10 I21 in the Swedish hospital discharge and cause-specific death registries) and mortality from ischaemic heart disease (defined as I20-I25 in the cause-specific death registry). Secondary endpoints were any major coronary event (defined as I21 or I20-I25 together with an operation code for PCI or CABG in the discharge registry or I20-I25 in the cause-specific death registry) and total mortality (defined as any registration in the cause-specific death registry).
Variables assessed were age, sex, diabetes duration, BMI, HbAlc (glycated haemoglobin), blood pressure, low-density lipoprotein and high-density lipoprotein cholesterol, triglycerides, renal function (creatinine), microalbuminuria, macroalbuminuria, smoking, level of education and antihypertensive or lipid-lowering medication use. BMI was calculated as body weight in kilograms divided by height in $\mathrm{m}^{2}$.

\section{STATISTICAL ANALYSIS}

The association between BMI and each endpoint was investigated using a separate proportional hazard regression model (Cox regression) for each endpoint. Several models were set up in a stepwise fashion adjusting for additional covariates in each step. Model 1 was adjusted for sex, age and diabetes duration. Model 2 was additionally adjusted for HbA1c, systolic and diastolic blood pressure and smoking. Model 3 was additionally adjusted for smoking, microalbuminuria and macroalbuminuria, lipid-lowering agents, blood pressure medications and level of education. Model 3 was the primary model. An additional model 4 was adjusted for the same covariates as model 3 but without macroalbuminuria and microalbuminuria and blood pressure. A separate analysis was performed to investigate the association between macroalbuminuria and BMI to analyse potential multicollinearity and overfitting.

The estimated HRs describe the relative risk increase of having a specific outcome in the patient population in each BMI group, with BMI $18.5-<25 \mathrm{~kg} / \mathrm{m}^{2}$ as the reference. For all models, the proportional hazards assumption was fulfilled.

For descriptive purposes, means with SDs are presented for continuous variables and numbers with percentages are presented for categorical variables

All calculations were performed with SAS V.9.4.

\section{RESULTS}

Among 17449 patients with type 1 diabetes, mean age was $39.5 \pm 13.2$ years, $55.0 \%$ were men and $45.0 \%$ women, mean diabetes duration was $24.0 \pm 13.7$ years and mean was BMI $25.2 \pm 3.8 \mathrm{~kg} / \mathrm{m}^{2}$. There were $819(4.7 \%)$ MI events (ICD-10 I21, first registration after inclusion) during a follow-up of 10 years, 276 (1.6\%) deaths from ischaemic heart disease (I20-I25) and 1261 (7.2\%) deaths. Baseline data for all groups are presented in table 1 , and all events are summarised and presented by BMI group in table 2 .

In model 1 (adjusted for sex, age and diabetes duration), there was an increase in risk for MI at all levels of overweight and obesity (BMI $>25 \mathrm{~kg} / \mathrm{m}^{2}$ ) (HR $1.21-$ 1.71) but not for mortality in ischaemic heart disease, where instead there was a significant increase in risk in the underweight group (BMI $<18.5 \mathrm{~kg} / \mathrm{m}^{2}$ ) (HR 3.30, 95\% CI 1.7-6.3). When additionally adjusted for HbA1c, blood pressure and smoking (model 2), the risk increase for MI disappeared in all groups except that with BMI $>35 \mathrm{~kg} / \mathrm{m}^{2}$ who still had a significantly increased risk 


\begin{tabular}{|c|c|c|c|c|c|c|}
\hline Variable & All $(n=17449)$ & $<18.5(n=182)$ & $\begin{array}{l}18.5-24.9 \\
(n=9140)\end{array}$ & $\begin{array}{l}25.0-29.9 \\
(n=6330)\end{array}$ & $\begin{array}{l}30.0-35.0 \\
(n=1454)\end{array}$ & $\begin{array}{l}>35.0 \\
(n=343)\end{array}$ \\
\hline Age, years (SD) & 39.5 (13.2) & $39.0(16.7)$ & $39.0(13.6)$ & $40.0(12.5)$ & $40.6(12.3)$ & $39.3(12.7)$ \\
\hline Diabetes duration, years (SD) & $24.0(13.7)$ & $23.2(17.5)$ & $23.8(14.1)$ & $24.5(13.1)$ & $24.0(13.2)$ & $20.7(13.9)$ \\
\hline BMI kg/m² (SD) & $25.2(3.8)$ & $17.7(0.8)$ & $22.6(1.6)$ & $27.0(1.4)$ & $31.8(1.4)$ & $38.2(3.7)$ \\
\hline Smokers, n (\%) & $2229(13.5)$ & $40(23.3)$ & $1319(15.2)$ & $658(11.0)$ & $183(13.4)$ & $29(9.3)$ \\
\hline Hba1c mmol/mol (SD) & $64.8(14.3)$ & $69.8(18.8)$ & $64.3(14.9)$ & $64.9(13.2)$ & $66.6(13.5)$ & $66.7(15.1)$ \\
\hline Systolic BP mm Hg (SD) & $128.4(16.8)$ & $123.2(20.8)$ & $126.5(16.7)$ & $129.9(16.3)$ & $133.4(16.7)$ & $130.4(17.4)$ \\
\hline Diastolic BP mm Hg (SD) & $73.9(8.8)$ & $71.1(9.3)$ & $72.8(8.7)$ & $74.8(8.7)$ & $76.9(9.0)$ & $76.6(9.2)$ \\
\hline LDL cholesterol mmol/L, (SD) & $2.8(0.8)$ & $2.6(0.8)$ & $2.7(0.8)$ & $2.9(0.8)$ & $3.0(0.9)$ & $3.0(0.9)$ \\
\hline HDL cholesterol mmol/L, (SD) & $1.6(0.5)$ & $1.7(0.4)$ & $1.7(0.5)$ & $1.6(0.4)$ & $1.5(0.4)$ & $1.3(0.4)$ \\
\hline Total cholesterol mmol/L, (SD) & $4.9(0.9)$ & $4.8(1.1)$ & $4.8(0.9)$ & $5.0(0.9)$ & $5.2(1.0)$ & $5.1(1.0)$ \\
\hline Triglycerides mmol/L, (SD) & $1.2(0.9)$ & $1.2(0.7)$ & $1.0(0.7)$ & $1.2(0.9)$ & $1.5(1.3)$ & $1.9(1.7)$ \\
\hline Creatinine $\mu \mathrm{mol} / \mathrm{L}$ (SD) & $87.7(49.8)$ & $94.3(72.1)$ & $87.6(52.9)$ & $87.6(41.4)$ & $89.6(60.2)$ & $83.6(46.9)$ \\
\hline Microalbuminuria, $n(\%)$ & 2127 (14.8) & $20(14.9)$ & 1014 (13.4) & $822(15.7)$ & $214(18.3)$ & $57(20.5)$ \\
\hline Macroalbuminuria, n (\%) & $1363(8.1)$ & $22(13.6)$ & $673(7.6)$ & $484(8.0)$ & $154(11.2)$ & $30(9.2)$ \\
\hline Primary school, n (\%) & 2494 (14.9) & $32(20.4)$ & 1169 (13.3) & $986(16.1)$ & $249(18.0)$ & $58(18.0)$ \\
\hline High school, n (\%) & 8449 (50.3) & $77(49.0)$ & 4191 (47.7) & 3210 (52.4) & $770(55.5)$ & $201(62.2)$ \\
\hline University, n (\%) & $5844(34.8)$ & $48(30.6)$ & 3430 (39.0) & 1934 (31.5) & $368(26.5)$ & $64(19.8)$ \\
\hline BP medication, n (\%) & 5002 (29.4) & $38(21.6)$ & $2336(26.1)$ & 1940 (31.5) & $553(39.4)$ & $135(40.1)$ \\
\hline Lipid medication, n (\%) & $2733(16.5)$ & $23(13.5)$ & $1124(12.9)$ & $1151(19.1)$ & $355(26.3)$ & $80(24.6)$ \\
\hline
\end{tabular}

BMI, body mass index; BP, blood pressure; HDL, high-density lipoprotein; LDL, low-density lipoprotein.

(HR $1.6995 \%$ CI 1.07-2.65). The additional risk in the underweight group (BMI $<18.5 \mathrm{~kg} / \mathrm{m}^{2}$ ) for mortality in ischaemic heart disease still remained in model 2 (HR $2.59,95 \%$ CI $1.35-5.0$ )

In Cox regression for the main model (model 3, adjusted for sex, age, diabetes duration, HbA1c, blood pressure (both diastolic and systolic), smoking, microalbuminuria and macroalbuminuria, lipid-lowering agents, blood pressure medications and level of education), there was no significant effect of BMI on the risk of MI or major coronary events (figures 1 and 2), with HRs ranging between 0.97 and 1.33 in model 3 and all including unity. However, there was a significant increased in risk of coronary death and all-cause mortality in the BMI $<18.5 \mathrm{~kg} / \mathrm{m}^{2}$ group (figures 3 and 4), with HRs of 5.0 (95\% CI 1.5 to 16.9) and 5.4 (95\% CI 3.1 to 9.6). After adjustment for known CVD risk factors, there was a statistically significant decrease in overall mortality in the overweight group (BMI $25-<30 \mathrm{~kg}$ / $\mathrm{m}^{2}$ ) when compared with normal weight (BMI 18.5$\left.24.9 \mathrm{~kg} / \mathrm{m}^{2}\right)$, with an HR of 0.72 (95\% CI 0.56 to 0.92$)$.

Baseline macroalbuminuria was a significant risk factor for all outcomes, with unadjusted HR ranging from 1.65 to 3.17 , whereas microalbuminuria showed no significant relationship for any event. No relationship between BMI and macroalbuminuria at baseline existed (correlation coefficient 0.02 ). In model 4 , which excluded renal complications and blood pressure, results were consistent with model 3.

Table 2 Crude incidence rate for endpoints (count (\%)) for 17449 patients with type 1 diabetes by categories of BMI

\begin{tabular}{|c|c|c|c|c|c|c|}
\hline Variable & All $(n=17449)$ & $<18.5(n=182)$ & $\begin{array}{l}18.5-24.9 \\
(n=9140)\end{array}$ & $\begin{array}{l}25.0-29.9 \\
(n=6330)\end{array}$ & $\begin{array}{l}30.0-35.0 \\
(n=1454) \\
\end{array}$ & $\begin{array}{l}>35.0 \\
(\mathrm{n}=343)\end{array}$ \\
\hline Myocardial infarction, $n$ (\%) & $819(4.7)$ & $15(8.2)$ & $386(4.2)$ & $316(5.0)$ & $81(5.6)$ & $21(6.1)$ \\
\hline Any major coronary event, $\mathrm{n}(\%)$ & $901(5.2)$ & $18(9.9)$ & $427(4.7)$ & $347(5.5)$ & $87(6.0)$ & $22(6.4)$ \\
\hline $\begin{array}{l}\text { Mortality in ischaemic heart disease, } \\
\mathrm{n}(\%)\end{array}$ & $276(1.6)$ & $10(5.5)$ & $127(1.4)$ & $105(1.7)$ & $28(1.9)$ & $6(1.7)$ \\
\hline Total mortality, n (\%) & $1261(7.2)$ & 50 (27.5) & $659(7.2)$ & $392(6.2)$ & $125(8.6)$ & $35(10.2)$ \\
\hline
\end{tabular}

BMI, body mass index. 


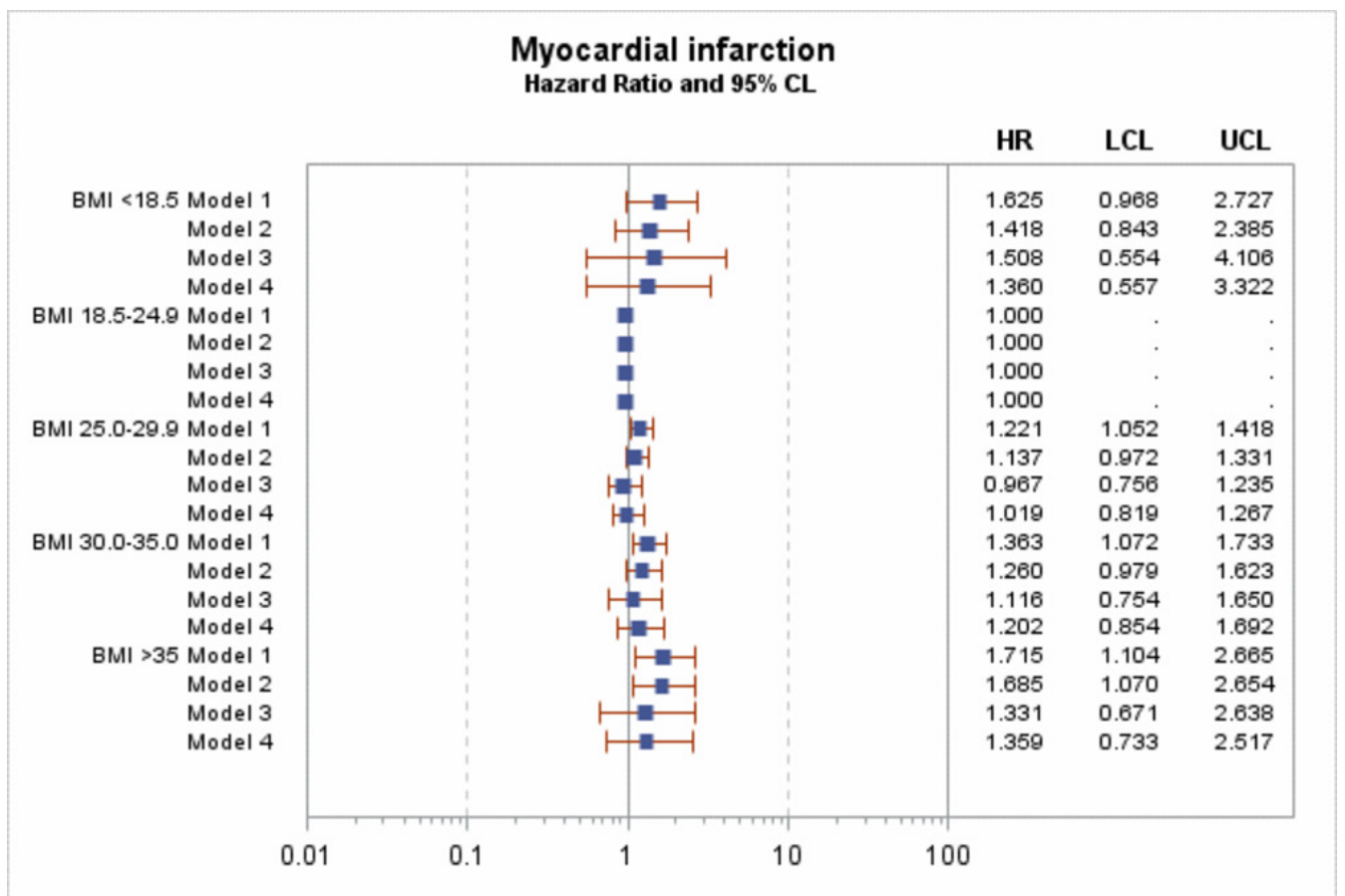

Figure $1 \mathrm{HR}$ for myocardial infarction. Model 1 is adjusted for sex, age and diabetes duration. Model 2 is adjusted for sex, age, diabetes duration $\mathrm{HbA1c}$, blood pressure (both diastolic and systolic) and smoking. Model 3 is adjusted for sex, age, diabetes duration, $\mathrm{HbA1c}$, blood pressure (both diastolic and systolic), smoking, microalbuminuria and macroalbuminuria, lipid-lowering agents, blood pressure medications and level of education. Model 4 is adjusted for sex, age, diabetes duration, HbA1c, smoking, lipid-lowering agents, blood pressure medications and level of education. BMI, body mass index. LCL (95\% Lower confidence limit) UCL (95\% Upper confidence limit)

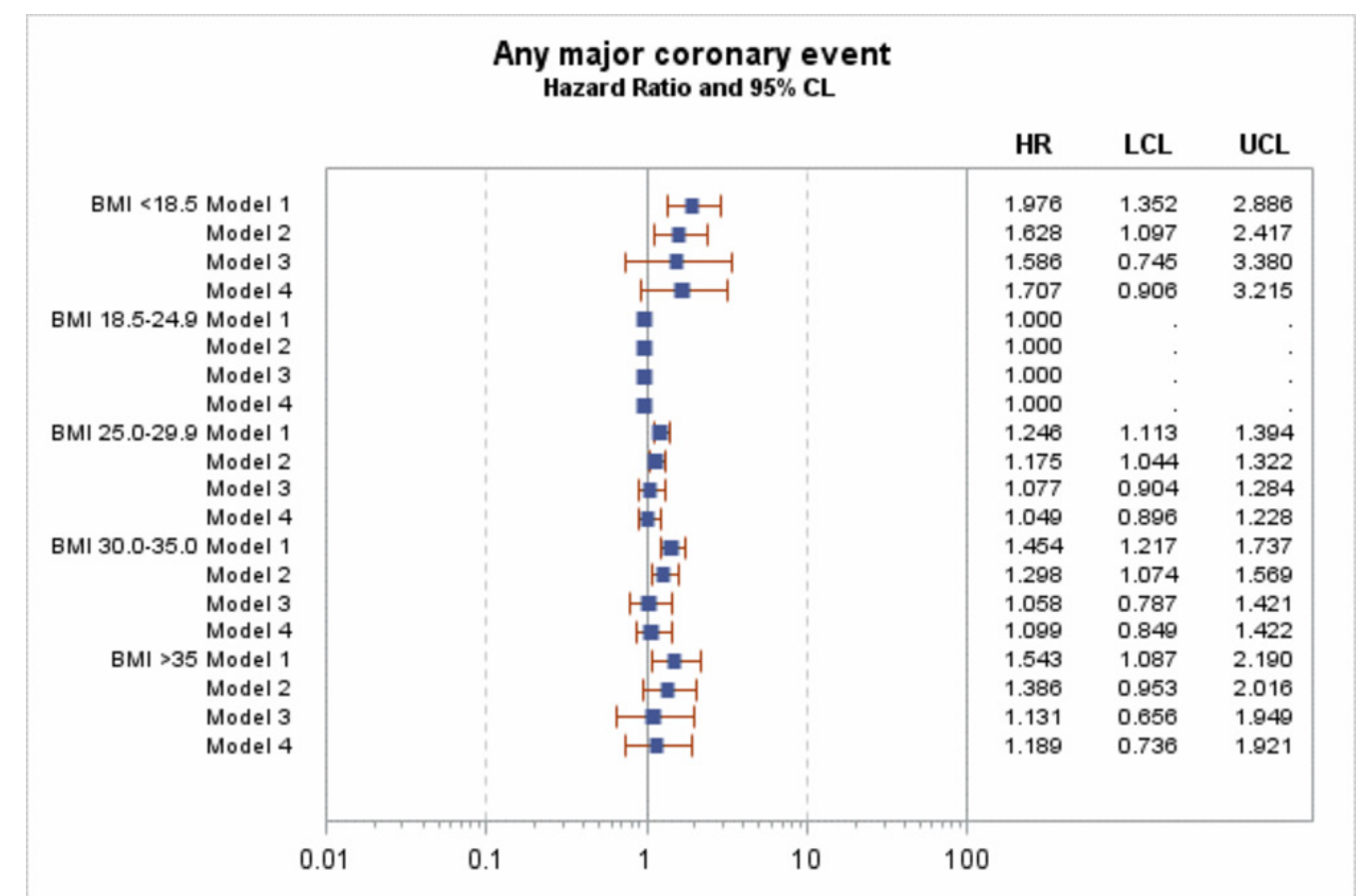

Figure 2 HR for any coronary event. Model 1 is adjusted for sex, age and diabetes duration. Model 2 is adjusted for sex, age, diabetes duration HbA1c, blood pressure (both diastolic and systolic) and smoking. Model 3 is adjusted for sex, age, diabetes duration, $\mathrm{HbA1c}$, blood pressure (both diastolic and systolic), smoking, microalbuminuria and macroalbuminuria, lipid-lowering agents, blood pressure medications and level of education. Model 4 is adjusted for sex, age, diabetes duration, HbA1c, smoking, lipid-lowering agents, blood pressure medications and level of education. BMI, body mass index. LCL (95\% Lower confidence limit) UCL (95\% Upper confidence limit) 


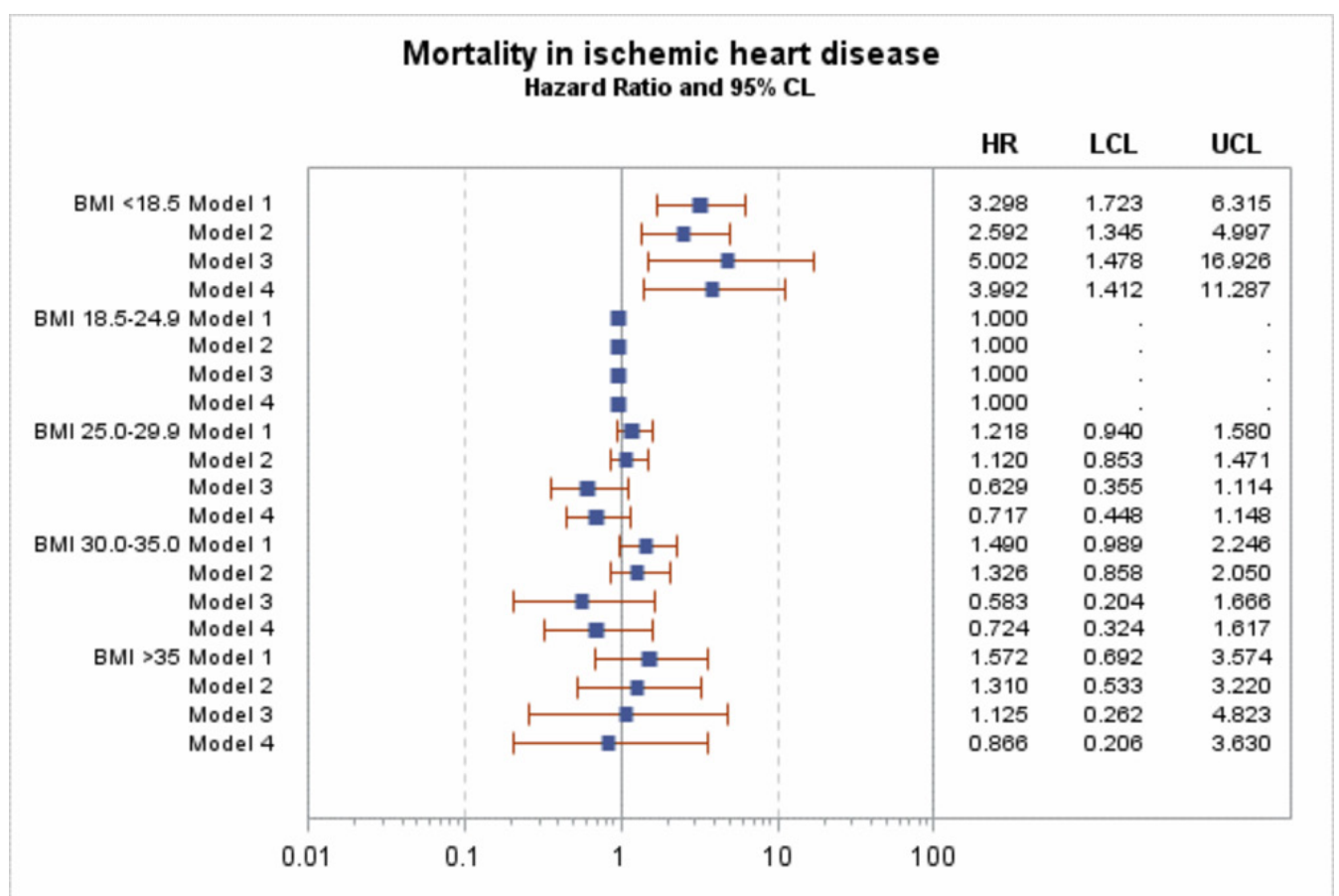

Figure 3 HR for mortality in ischemic heart disease. Model 1 is adjusted for sex, age and diabetes duration. Model 2 is adjusted for sex, age, diabetes duration $\mathrm{HbA1c}$, blood pressure (both diastolic and systolic) and smoking. Model 3 is adjusted for sex, age, diabetes duration, $\mathrm{HbA1c}$, blood pressure (both diastolic and systolic), smoking, microalbuminuria and macroalbuminuria, lipid-lowering agents, blood pressure medications and level of education. Model 4 is adjusted for sex, age, diabetes duration, $\mathrm{HbA1c}$, smoking, lipid-lowering agents, blood pressure medications and level of education. BMI, body mass index. LCL (95\% Lower confidence limit) UCL (95\% Upper confidence limit)

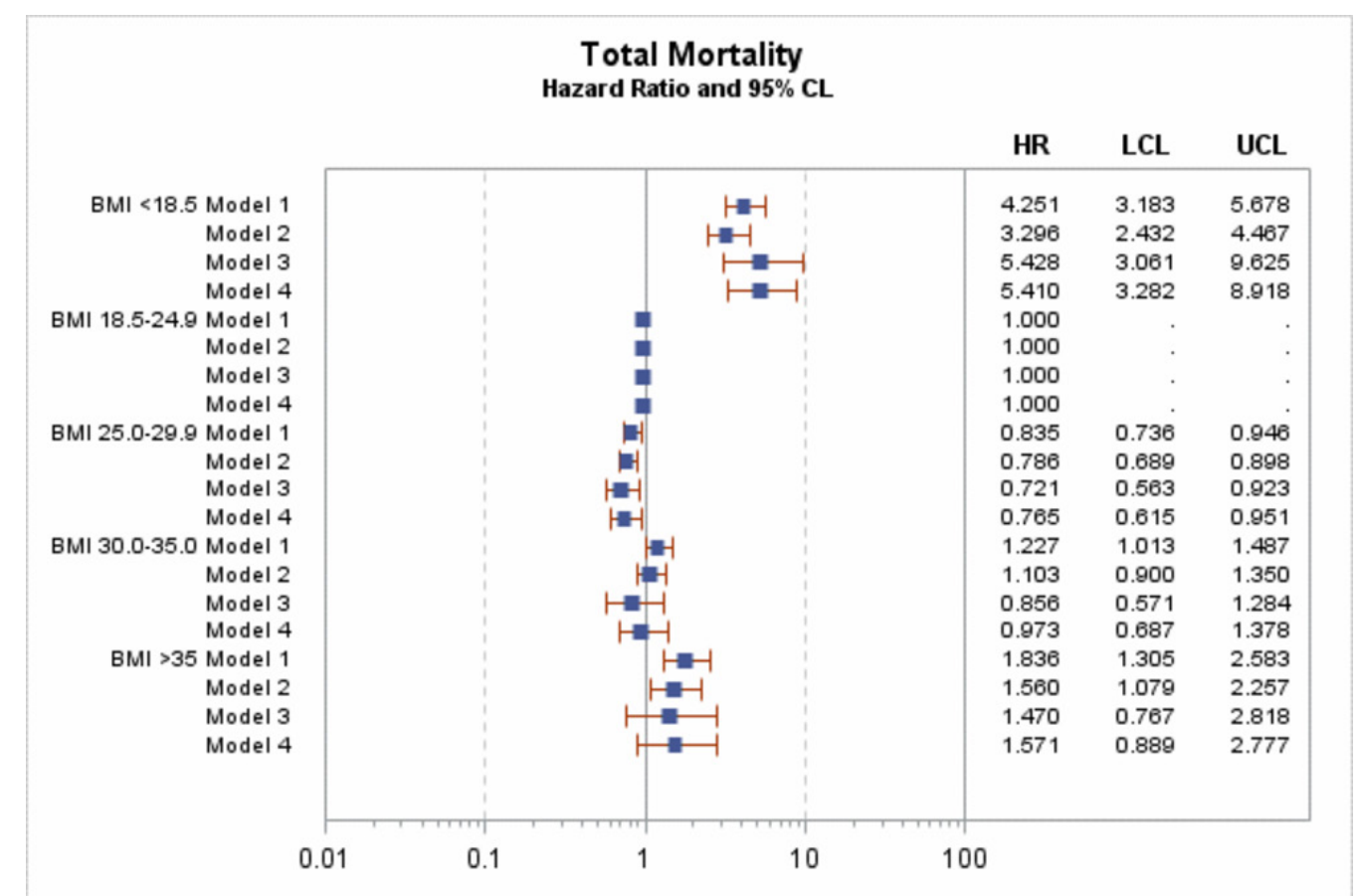

Figure $4 \mathrm{HR}$ for total mortality. Model 1 is adjusted for sex, age and diabetes duration. Model 2 is adjusted for sex, age, diabetes duration HbA1c, blood pressure (both diastolic and systolic) and smoking. Model 3 is adjusted for sex, age, diabetes duration, $\mathrm{HbA1c}$, blood pressure (both diastolic and systolic), smoking, microalbuminuria and macroalbuminuria, lipid-lowering agents, blood pressure medications and level of education. Model 4 is adjusted for sex, age, diabetes duration, $\mathrm{HbA1c}$, smoking, lipid-lowering agents, blood pressure medications and level of education. BMI, body mass index. LCL (95\% Lower confidence limit) UCL (95\% Upper confidence limit) 


\section{DISCUSSION}

In this population-based study of 17436 patients with type 1 diabetes, BMI had no independent impact on the risk for MI or major coronary events, but there was a statistically significant increase in risk for death due to either cardiovascular or all causes in the group with low BMI $<18.5 \mathrm{~kg} / \mathrm{m}^{2}$ compared with normal weight (BMI $18.5-25 \mathrm{~kg} / \mathrm{m}^{2}$ ).

A previous meta-analysis based on many general population cohorts $^{21}$ showed a statistically significant HR for $\mathrm{BMI}$ as a risk factor for coronary heart disease only in patients with BMI over $30 \mathrm{~kg} / \mathrm{m}^{2}$ in a model adjusted for blood pressure, lipids and diabetes. Other studies on obesity and all-cause mortality have shown J-shaped or U-shaped relationships in both type 1 diabetes and the general population, ${ }^{22}{ }^{23}$ with increased mortality in the underweight group. The obesity paradox, referring to a protective effect with overweight and moderate obesity, may be an explanation for decreased total mortality in the group with BMI $25-30 \mathrm{~kg} / \mathrm{m}^{2}$. The reason for this effect seen in many different diseases is still under discussion ${ }^{15}$ but may be related to the endocrine effect of adipose tissue.

The mechanism behind the increased risk of CVD among patients with type 1 diabetes is most likely multifactorial. Several different mechanisms have been proposed. $^{24}$ Among these are oxidative stress from hyperglycaemia and cardiac autonomic neuropathy. We have previously shown that both heart failure ${ }^{2}$ and $\mathrm{MI}^{6}$ are substantially more common in patients with type 1 diabetes than the general population of a similar magnitude with an excess risk of around 4.

Increased BMI has been shown to be a strong risk factor for development of heart failure, ${ }^{18}$ with a $50 \%$ risk increase in obesity (BMI $30-35 \mathrm{~kg} / \mathrm{m}^{2}$ ). According to our results, obesity does not seem to have the same effect on coronary disease, implying there may be different mechanisms involved in the development of heart failure and coronary disease. In the development of heart failure, glucose toxicity (indicated by a risk increase with higher levels of $\mathrm{HbA1c}$ ) seems to be an important risk factor, with a risk increase of 1.26-3.98 with increasing updated mean HbA1c. ${ }^{25}$ A possible mechanism may be that higher BMI changes glucose metabolism in a negative way increasing toxic effects on the cardiomyocyte. This mechanism is probably not present in coronary disease, as we did not see a strong relation between increased risk for MI and BMI.

A clear excess risk of mortality still exists in patients with type 1 diabetes compared with the general population, ${ }^{7}$ where high HbAlc and renal complications have been strong risk factors. One essential aim of diabetes care is to continue lowering this excess risk to that of the general population. Although the current data are observational and should be interpreted with caution, they indicate that overweight and obesity are not the main factors driving excess mortality. However, low BMI $\left(<18.5 \mathrm{~kg} / \mathrm{m}^{2}\right)$ was a risk factor. This may indicate that these patients have other comorbidities or risk factors not possible to control for here rather than that low BMI per se leads to high risk. In any case, clinicians should be aware that low BMI is a risk factor for mortality.

Even if drawing conclusions regarding causality from observational data may be problematic, our results imply that there are different pathways for the increased risk of developing coronary heart disease and heart failure. One could speculate that while BMI is still an important risk factor for coronary heart disease among patients without type 1 diabetes, the mechanism may be similar to the effects of type 1 diabetes on the coronary arteries and, therefore, not additive among patients with type 1 diabetes. Maintaining a healthy weight among patients with type 1 diabetes should still be a priority; however, obesity is still an important risk factor for many other diseases and generally increases insulin resistance, something that makes it harder for persons with type 1 diabetes to adjust their insulin dosing.

Strengths of this study include a relatively large and unselected population with type 1 diabetes and adjustment for many variables such as diabetes duration and level of education. Limitations include the reliance on hospital discharge data and the cause-specific death registry for all endpoints. However, acute MI has been validated in the hospital discharge registry ${ }^{26}$ with a validity of $91 \%$, while the validity of diagnoses in the cause specific death registry is less. Johansson et $a l^{27}$ found an agreement of $83 \%$ between last main diagnosis and cause of death in hospitals but only $43 \%$ agreement in non-hospital deaths. Additionally, since our study was observational, it is not possible to draw causal conclusions.

In conclusion, BMI did not seem to have any impact on risk of MI in this group of persons with type 1 diabetes, possibly due to treatment of secondary risk factors such as hypertension and lipid abnormalities. The different effect of BMI on the risk of developing ischaemic heart disease compared with heart failure in a similar population suggests different mechanisms behind the diseases. Even if we did not observe an increased risk for CVD, there is still an important role for weight management in type 1 diabetes, as obesity increases both insulin resistance $^{28}$ and risk of heart failure. ${ }^{18}$

Acknowledgements We want to thank all data collecting clinicians and staff at the National Diabetes Registry. Editorial assistance was provided by Joseph W Murphy.

Contributors DV, AR, KE-0, A-MS and ML designed the study protocol. MM and SF designed and did the statistical analyses. DV and ML wrote the first draft. All authors participated in revising and rewriting the manuscript and approved the final version.

Funding The study was funded by grants from Novo Nordisk foundation and the Swedish state, under an agreement between the Swedish national government and the county councils concerning economic support for research and education of doctors (ALF-agreement).

Competing interests None declared.

Ethics approval The study was approved by the regional ethics review board at the University of Gothenburg (Sweden). 
Provenance and peer review Not commissioned; externally peer reviewed.

Data sharing statement No additional data are available.

Open Access This is an Open Access article distributed in accordance with the Creative Commons Attribution Non Commercial (CC BY-NC 4.0) license, which permits others to distribute, remix, adapt, build upon this work non-commercially, and license their derivative works on different terms, provided the original work is properly cited and the use is non-commercial. See: http://creativecommons.org/ licenses/by-nc/4.0/

(C) Article author(s) (or their employer(s) unless otherwise stated in the text of the article) 2018. All rights reserved. No commercial use is permitted unless otherwise expressly granted.

\section{REFERENCES}

1. Wilkins E, Wilson L, Wickramasinghe K, et al. European cardiovascular disease statistics 2017. Brussels: European Heart Network, 2017.

2. Rosengren A, Vestberg D, Svensson AM, et al. Long-term excess risk of heart failure in people with type 1 diabetes: a prospective case-control study. Lancet Diabetes Endocrinol 2015;3:876-85.

3. Laing SP, Swerdlow AJ, Slater SD, et al. Mortality from heart disease in a cohort of 23,000 patients with insulin-treated diabetes. Diabetologia 2003;46:760-5.

4. Dorman JS, Laporte RE, Kuller LH, et al. The Pittsburgh insulindependent diabetes mellitus (IDDM) morbidity and mortality study. Mortality results. Diabetes 1984;33:271-6.

5. Rawshani A, Rawshani A, Franzén S, et al. Range of risk factor levels: control, mortality, and cardiovascular outcomes in type 1 diabetes mellitus. Circulation 2017;135:1522-31.

6. Matuleviciene-Anängen V, Rosengren A, Svensson AM, et al. Glycaemic control and excess risk of major coronary events in persons with type 1 diabetes. Heart 2017;103:1687-95. Epub ahead of print.

7. Rawshani A, Rawshani A, Franzén S, et al. Mortality and cardiovascular disease in type 1 and type 2 diabetes. N Engl J Med 2017;376:1407-18.

8. Lind M, Svensson AM, Kosiborod M, et al. Glycemic control and excess mortality in type 1 diabetes. $N$ Engl J Med 2014;371:1972-82.

9. Ahlén E, Pivodic A, Wedel H, et al. Glycemic control, renal complications, and current smoking in relation to excess risk of mortality in persons with type 1 diabetes. J Diabetes Sci Technol 2016;10:1006-14

10. Go AS, Mozaffarian D, Roger VL, et al. Heart disease and stroke statistics--2013 update: a report from the American Heart Association. Circulation 2013;127:e6-e245.

11. Soedamah-Muthu SS, Chaturvedi N, Toeller M, et al. Risk factors for coronary heart disease in type 1 diabetic patients in Europe: the EURODIAB Prospective Complications Study. Diabetes Care 2004;27:530-7.
12. Nathan DM, Cleary PA, Backlund JY, et al. Intensive diabetes treatment and cardiovascular disease in patients with type 1 diabetes. N Engl J Med 2005;353:2643-53.

13. Eeg-Olofsson K, Cederholm J, Nilsson PM, et al. Glycemic control and cardiovascular disease in 7,454 patients with type 1 diabetes: an observational study from the Swedish National Diabetes Register (NDR). Diabetes Care 2010;33:1640-6.

14. Whitlock G, Lewington S, Sherliker P, et al. Body-mass index and cause-specific mortality in 900000 adults: collaborative analyses of 57 prospective studies. Lancet 2009;373:1083-96.

15. Angerås $\mathrm{O}$, Albertsson $\mathrm{P}, \mathrm{Karason} \mathrm{K}$, et al. Evidence for obesity paradox in patients with acute coronary syndromes: a report from the Swedish coronary angiography and angioplasty registry. Eur Heart J 2013;34:345-53.

16. De Schutter A, Lavie CJ, Milani RV. The impact of obesity on risk factors and prevalence and prognosis of coronary heart disease-the obesity paradox. Prog Cardiovasc Dis 2014:56:401-8.

17. Di Angelantonio E, Bhupathiraju S, Global BMI Mortality Collaboration. Body-mass index and all-cause mortality: individualparticipant-data meta-analysis of 239 prospective studies in four continents. Lancet 2016;388:776-86.

18. Vestberg D, Rosengren A, Olsson M, et al. Relationship between overweight and obesity with hospitalization for heart failure in 20,985 patients with type 1 diabetes: a population-based study from the Swedish National Diabetes Registry. Diabetes Care 2013;36:2857-61.

19. Olén O, Bihagen E, Rasmussen F, et al. Socioeconomic position and education in patients with coeliac disease. Dig Liver Dis 2012;44:471-6.

20. Emilsson L, Smith JG, West J, et al. Increased risk of atrial fibrillation in patients with coeliac disease: a nationwide cohort study. Eur Heart J 2011;32:2430-7.

21. Wormser D, Kaptoge S, Di Angelantonio E, et al. Separate and combined associations of body-mass index and abdominal adiposity with cardiovascular disease: collaborative analysis of 58 prospective studies. Lancet 2011;377:1085-95.

22. Calle EE, Thun MJ, Petrelli JM, et al. Body-mass index and mortality in a prospective cohort of U.S. adults. N Engl J Med 1999;341:1097-105.

23. Conway B, Miller RG, Costacou T, et al. Adiposity and mortality in type 1 diabetes. Int J Obes 2009;33:796-805.

24. Schnell O, Cappuccio F, Genovese S, et al. Type 1 diabetes and cardiovascular disease. Cardiovasc Diabetol 2013;12:156.

25. Lind M, Bounias I, Olsson M, et al. Glycaemic control and incidence of heart failure in 20,985 patients with type 1 diabetes: an observational study. Lancet 2011;378:140-6.

26. Elo SL, Karlberg IH. Validity and utilization of epidemiological data: a study of ischaemic heart disease and coronary risk factors in a local population. Public Health 2009;123:52-7.

27. Johansson LA, Westerling R. Comparing Swedish hospital discharge records with death certificates: implications for mortality statistics. Int J Epidemiol 2000;29:495-502.

28. Reinehr T, Holl RW, Roth CL, et al. Insulin resistance in children and adolescents with type 1 diabetes mellitus: relation to obesity. Pediatr Diabetes 2005;6:5-12. 